\title{
On the Techniques to Design 3D-Printable Arbitrarily Height-Modulated Substrate Integrated Waveguide Filters
}

\author{
Leticia Alonso-González* \\ Dept. of Electrical Engineering. \\ University of Oviedo. \\ Gijón, Spain. \\ leti.algo@gmail.com
}

\author{
Samuel Ver-Hoeye \\ Dept. of Electrical Engineering. \\ University of Oviedo. \\ Gijón, Spain. \\ versamuel@uniovi.es
}

\author{
Miguel Fernández-García \\ Dept. of Electrical Engineering. \\ University of Oviedo. \\ Gijón, Spain. \\ fernandezgmiguel@uniovi.es
}

\author{
Carlos Vázquez-Antuña \\ Dept. of Electrical Engineering. \\ University of Oviedo. \\ Gijón, Spain. \\ vazquezcarlos@uniovi.es
}

\author{
Fernando Las-Heras Andrés \\ Dept. of Electrical Engineering. \\ University of Oviedo. \\ Gijón, Spain. \\ flasheras@uniovi.es
}

\begin{abstract}
A novel modelling technique to design microwave 3D-printable filters based on arbitrarily height-modulated substrate integrated waveguides (SIW) is presented in this paper. In the literature, different topologies have been already employed to design filters based on SIW. Nevertheless, none of these solutions have analyzed the behavior of the modulation of the height of the filters. This is due to the difficulty of manufacturing the prototypes with conventional procedures. In this paper, the techniques to design and manufacture these filters, provided with microstrip transitions for their validation, are presented.
\end{abstract}

Index Terms-3D-printing, filter, modelling, simulations, substrate integrated waveguide (SIW)

\section{INTRODUCTION}

In the literature, different topologies have been already employed to design filters based on SIW [1], [2]. Nevertheless, none of these solutions have analyzed the behavior of the modulation of the height of the filters. The herein presented technique is a parametric modelling to develop arbitrarily height-modulated substrate integrated waveguide (AHMSIW) filters. Although the conventional fabrication of a heightmodulated SIW using standard prototyping machinery represents a complex problem, due to the difficulty of achieving different heights in a conventional substrate, in this paper a novel alternative based on 3D-printing techniques is proposed.

In order to manufacture a height-modulated SIW structure using conventional techniques, one could use a milling machine and subsequently provide the substrate with the conductive vias and the required electrical conductivity through the

This work has been supported by the Spanish Agencia Estatal de Investigación (AEI) and Fondo Europeo de Desarrollo Regional (FEDER) under projects TEC2016-80815-P (AEI/FEDER-UE) and TEC2015-72110EXP (AEI), and FPU14/00016 grant, and by the Gobierno del Principado de Asturias (PCTI/FEDER-FSE) under projects IDI/2016/000372 and IDI/2017/000083. deposition of a thin gold layer using a sputtering process. Alternatively, the height-modulated SIW filter could be also manufactured using various stacked layers of substrate segments to achieve the different heights, avoiding the use of a milling machine. In order to connect the different layers, a gluing procedure could be employed, although the alignment of the different segments which compose the multilayered substrate and corresponding vias represents a challenge. Due to the beforementioned difficulties, in this paper, a new alternative based on 3D-printing procedures is proposed.

The work is organised as follows. In Section II, the modelling techniques are extensively explained and analysed. In Section III, two different examples are presented. In Section $\mathrm{IV}$, the fabrication procedure is discussed.

\section{Modelling}

\section{A. Description}

An AHMSIW is a SIW structure whose height discretly changes along its propagation direction in order to obtain a certain frequency response. For this purpose, the length of the SIW has been divided into $N$ segments and two different heights, $H_{1}$ and $H_{2}$, have been defined. Note that the number of different heights can be more than two, although in this work, the simplest version of the modelling is presented and analysed.

The separation between the inner rows of conductive vias of the SIW has been denoted by $W_{\text {SIW }}$, as depicted in Figure 1. The length of each segment in which the SIW has been divided is denoted by $L_{i}$, with $i \in[1, N]$. Each length, $L_{i}$, can be optimised in order to achieve a required bandstop or bandpass response of the filter. Additionally, the dielectric characteristics of the substrate, which can be either air or a different and lossy one, are represented as $\varepsilon_{\mathrm{eq}}$ and $\tan (\delta)_{\mathrm{eq}}$, for the relative 


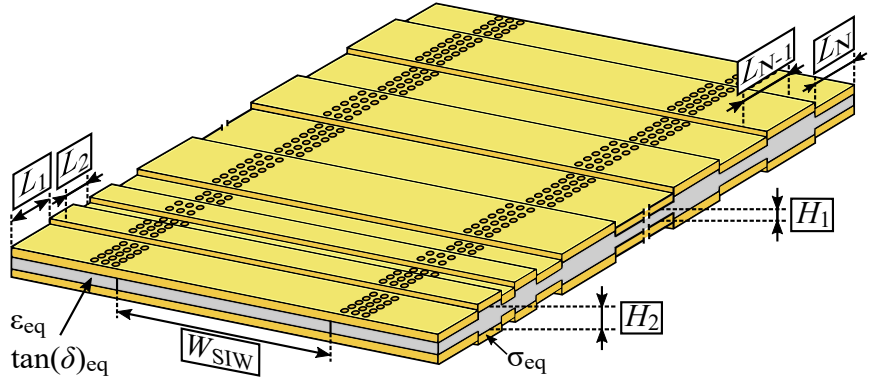

Fig. 1. Overview of a generic AHMSIW filter.

dielectric permittivity and the loss tangent, respectively. The electrical conductivity of the metallic elements of the structure is represented using $\sigma_{\text {eq }}$.

\section{B. Implementation}

In order to implement the filters, four different blocks have been defined for their posterior electromagnetic (EM) simulation. These blocks are a $H_{1}$-height SIW segment, a $H_{2}$ height SIW segment, a stepped SIW segment whose height is modified along its propagation direction from $H_{1}$ to $H_{2}$, and a stepped SIW segment whose height is modified from $\mathrm{H}_{2}$ to $H_{1}$, respectively, as depicted in Figure 2. In order to achieve a structure as the one depicted in Figure 1, the different blocks have to be connected in a precise order, as schematically represented in Figure 3.

The blocks denoted by $H_{1 \_2 \_B l o c k}$ and $H_{2 \_1 \_B l o c k}$ can be EM simulated given their dielectric parameters, $\varepsilon_{\mathrm{eq}}$ and $\tan (\delta)_{\mathrm{eq}}$, conductivity, $\sigma_{\mathrm{eq}}$, and dimensions, using the 3-D full-wave simulation software ANSYS HFSS, High Frequency Structure Simulator, and obtaining their scattering parameters $(S$ parameters). Likewise, $H_{1 \_ \text {Block }}$ and $H_{2 \_ \text {Block }}$ blocks are simulated using HFSS given their dielectric parameters, conductivity, dimensions, and sweeping their length parameters, $L 1$ and $L 2$, in order to obtain the different $S$-parameters datasets as a function of the length. As an example, $H_{1 \_ \text {Block }}$ block is simulated for different values of its length, $L 1 \in[1,30]$ $\mathrm{mm}$. As a result of each simulation, the $S$-parameters are exported into a Touchstone S2P, 2-port S-parameter, file as depicted in Figure $4 \mathrm{a}$. Once every dataset of $S$-parameters has been exported, then a MultiDimensional File, MDIF file, can be generated by defining the sweep variable before the corresponding $S$-parameters. In Figure $4 \mathrm{~b}$, the variable $p_{-}$filter has been defined, whose value corresponds to the length of the SIW segment in millimetres. Two MDIF files, associated to the $S$-parameters of the $H_{1 \_ \text {Block }}$ and $H_{2 \_ \text {Block }}$ blocks, have been generated and named $f_{-} h 1 . m d f$ and $f_{-} h 2 . m d f$, respectively. Similarly, another MDIF file, associated to the $S$-parameters of both, the $H_{1 \_2}$ Block and $H_{2 \_}{ }_{-}$Block blocks, has been generated and named $f_{-} h 12 . m d f$.

A schematic model of the AHMSIW filter can be designed using Advanced Design System, ADS, once the three MDIF files have been created, as summarised in Figure 5. Figure 5 a depicts the concatenation of the different defined blocks.

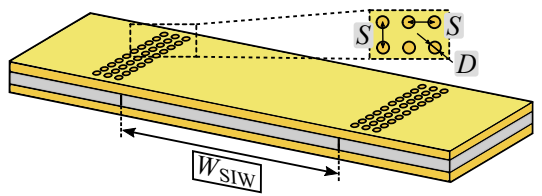

(a)

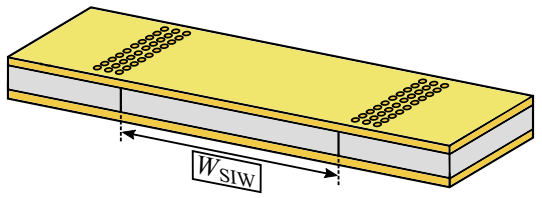

(c)

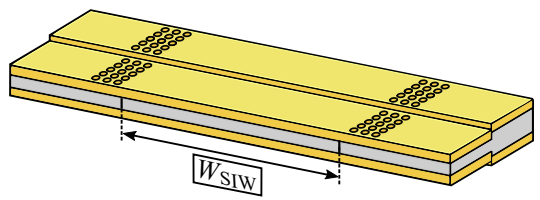

(e)

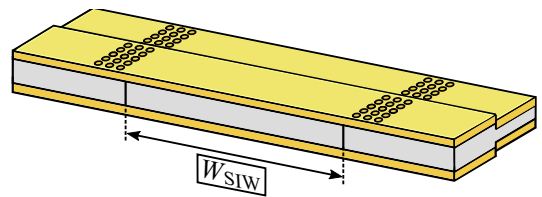

(g)

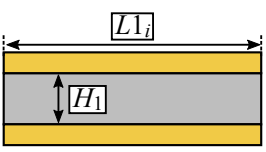

(b)

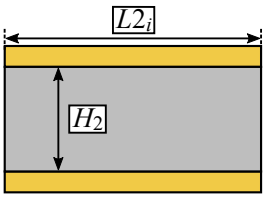

(d)

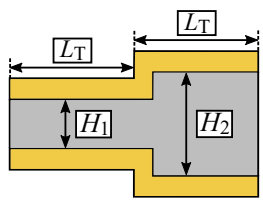

(f)

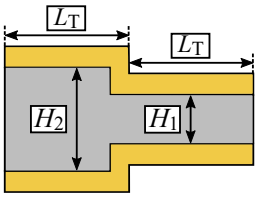

(h)
Fig. 2. Different blocks involved in the filter design (left: overview, right: side view). (a) $H_{1 \_B l o c k}$ block. (b) $H_{1 \_B l o c k}$ block. (c) $H_{2}$ Block block. (d) $H_{2}$ Bblock block. (e) $\bar{H}_{1 \_2 \_B l o c k}$ block. (f) $\bar{H}_{1 \_2 \_B l o c k}$ block. (g) $H_{2 \_1 \_B l o c k}$ block. (h) $H_{2} \_1 \_$Block block.

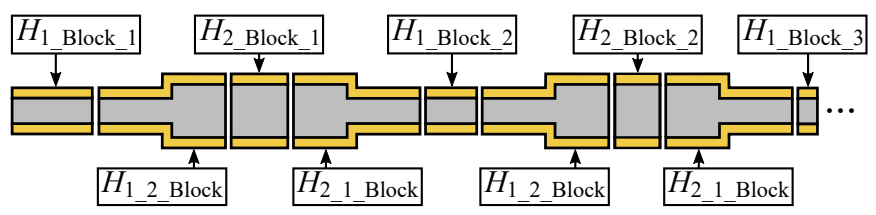

Fig. 3. Side view of the connection between the blocks to achieve bandstop or bandpass AHMSIW filters.

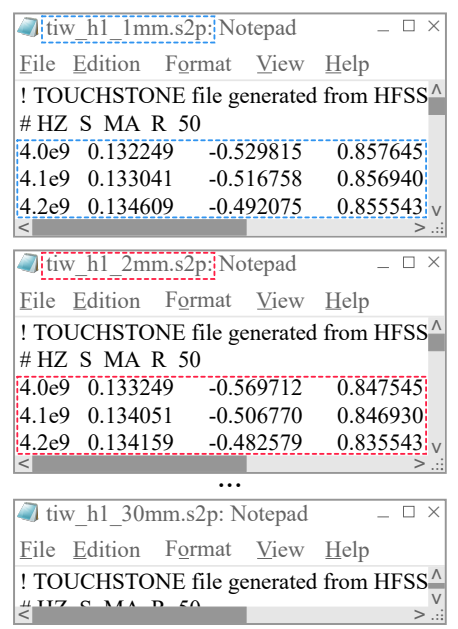

(a)

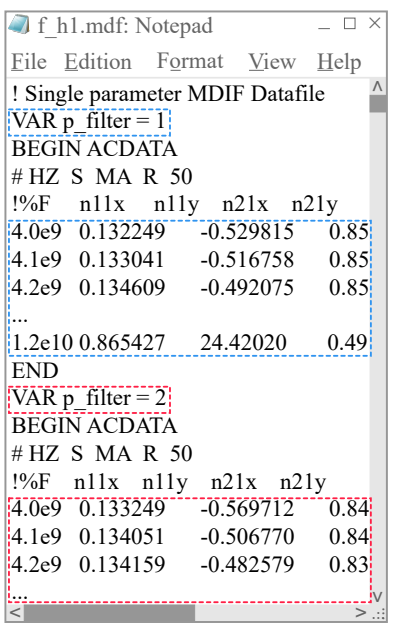

(b)
Fig. 4. Simulated $S$-parameters and corresponding files. (a) Different Touchstone S2P files for each length sweep. (b) Generated MDIF file. 


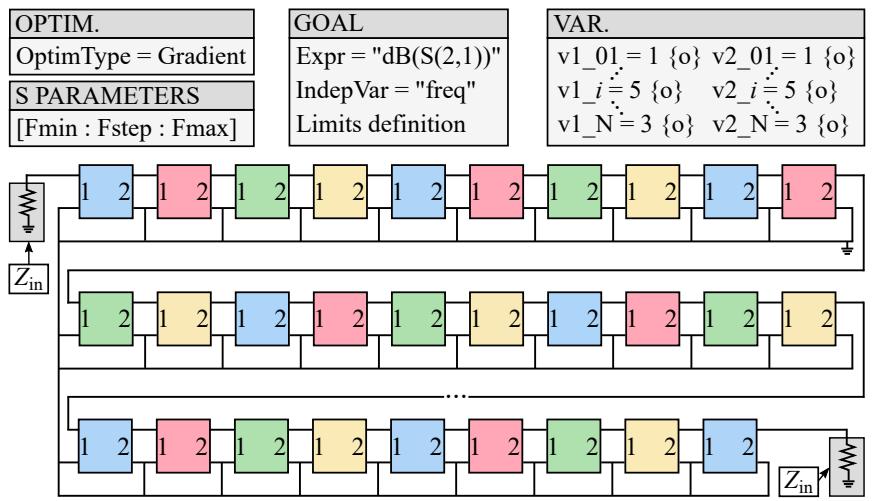

(a)

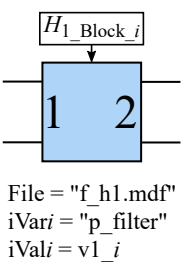

(b)

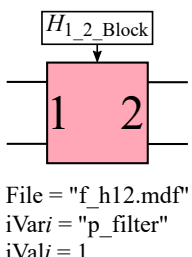

(c)

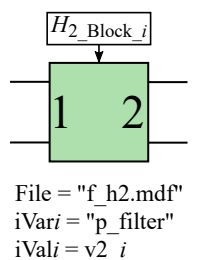

(d)

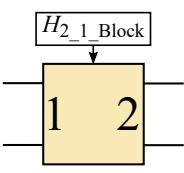

File $=$ "f_h12.mdf" iVar $i=$ "p filter" $\mathrm{iVal} i=2$

(e)
Fig. 5. Schematic model of the AHMSIW filter optimisation using ADS. (a) Schematic model outline. (b) Generic $H_{1 \_B l o c k}$ block. (c) Generic $H_{1}{ }_{2}$ _Block

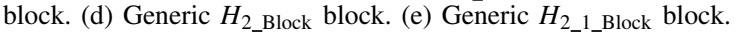

Connected to the first and last block of the concatenation, there are two identical terminals whose characteristic impedance, $Z_{\text {in }}$, is given by (1) [3], where $D$ and $S$ are the diameter and the separation between two consecutive conductive vias of the SIW, respectively.

$$
Z_{\text {in }}=\frac{2 \pi f \mu_{0}}{\sqrt{\left(\frac{2 \pi f}{c / \sqrt{\varepsilon_{\mathrm{eq}}}}\right)^{2}-\left(\frac{\pi}{W_{S I W}-\frac{S^{2}}{0.95 S}}\right)^{2}}}
$$

Figure $5 \mathrm{~b}$ depicts $i$-th $H_{1}$ Block block associated to the $f \_h l . m d f$ file. Therefore, the value of $p \_$filter is equal to $\mathrm{v} 1 \_i$, being $\mathrm{v} 1 \_i$ a variable to optimise. Figure 5c depicts the $H_{1 \_2 \_B l o c k}$ block associated to the $f \_h 12 . m d f$ file. Consequently, the value of $p_{-}$filter is 1 , due to the fact that in this file, there are only the datasets associated to the $H_{1 \_2 \_B l o c k}$ and $H_{2}{ }_{1}$ B Block blocks. Figure $5 \mathrm{~d}$ depicts $i$-th $H_{2}$ Block block associated to the $f \_h 2 . m d f$ file. Therefore, the value of $p \_$filter is equal to $\mathrm{v} 2 \_i$, being $\mathrm{v} 2 \_i$ a variable to optimise. Figure 5e depicts the $H_{2}$ 1_Block block associated to the $f_{-} h 12 . m d f$ file. Consequently, the value of $p$ filter is 2 . In other words, the variable $p$ filter indicates the value of the corresponding optimised variable for the blocks $H_{1 \_ \text {Block_ } i}$ or $H_{2 \_ \text {Block_ } i}$, whereas it indicates 1 or 2 for the transition blocks $H_{1 \_2 \_B l o c k}$ or $H_{2} \_$_B Block, respectively.

Once the schematic model is configured in ADS, an optimisation of the $\mathrm{v} 1 \_i$ and $\mathrm{v} 2 \_i$ variables can be carried out in order to obtain the required response of the filter. An optimisation based on the gradient can be used, although at the end of the optimisation, the values of v1_i and v2 $i$ are not integers, leading to the interpolation of the $S$-parameters. For

\begin{tabular}{|c|c|c|c|c|c|}
\hline Simulate & Export & Generate & Optimise & Truncate & Tune \\
\hline $\begin{array}{c}S \text {-parameters } \\
\text { using HFSS }\end{array}$ & $\begin{array}{l}\text { S2P } \\
\text { files }\end{array}$ & $\begin{array}{c}\text { MDIF } \\
\text { files }\end{array}$ & $\begin{array}{l}\mathrm{v} 1 \_i, \mathrm{v} 2 \_i \\
\text { variables }\end{array}$ & $\begin{array}{l}\text { v1_i, v2_i } \\
\text { variables }\end{array}$ & $\begin{array}{l}\mathrm{v} 1 \_i, \mathrm{v} 2 \_i \\
\text { variables }\end{array}$ \\
\hline
\end{tabular}

Fig. 6. Block diagram to summarise the AHMSIW design.

this reason, the values of $\mathrm{v} 1 \_i$ and $\mathrm{v} 2 \_i$ can be truncated and a tuning process may be required, as summarised in Figure 6. Although ADS implements a discrete optimiser, it cannot be applied to such a complex optimisation problem, due to the associated difficulty to converge.

\section{EXAMPLES}

With the aim of demonstrating the validity of the modelling already presented, a bandpass arbitrarily height-modulated substrate integrated waveguide filter (BPAHMSIW) and a bandstop arbitrarily height-modulated substrate integrated waveguide filter (BSAHMSIW) are presented in this section.

\section{A. Design of a BPAHMSIW filter}

A BPAHMSIW filter, whose passband belongs to the range of frequencies between 8 and $9 \mathrm{GHz}$, is proposed. The filter has been designed using the procedure thoroughly explained in section II and, for this purpose, the filter is composed of 61 blocks.

The blocks employed for the development of the BPAHMSIW filter are the four ones already explained in the previous section, whose thickness of the conductive layers has been denoted by $T$. In order to discretely modulate the height of the SIW, two different values for the height are defined, $H_{1}$ and $H_{2}$, respectively. The dimensions are summarised in Table I. The electrical parameters have been chosen to be: $\varepsilon_{\mathrm{eq}}=1.7$, $\tan (\delta)_{\mathrm{eq}}=0.00362$ and $\sigma_{\mathrm{eq}}=5 \cdot 10^{5} \mathrm{~S} / \mathrm{m}$

Figure 7 depicts the $\left|S_{11}\right|$ parameters, and detailed magnifications, corresponding to different lengths of the $H_{1}$ Block block. Figure 8 represents the corresponding $\left|S_{21}\right|$ parameters associated to the same lengths, for the $H_{1}$ Bblock blocks. Analogously, Figure 9 and Figure 10 represent the beforementioned data, but associated to the $H_{2}$ Block blocks.

Although the $H_{1}$ Block and $H_{2}$ Block blocks have been simulated using lengths from $0.5 \mathrm{~mm}$ to $15 \mathrm{~mm}$ with a step length of $0.5 \mathrm{~mm}$, in the graphics only half of the simulations have been included for simplification. The transition blocks have also been simulated as depicted in Figure 11. Once the different blocks have been simulated and the Touchstone S2P files are obtained, the three MDIF files are generated as previously explained in Figure 4.

The schematic model in ADS can be implemented once the MDIF files have been generated. For this purpose, a

TABLE I

DIMENSIONS OF THE SIW STRUCTURE OF THE BLOCKS.

\begin{tabular}{cccccccc}
\hline Dimension & $W_{\text {SIW }}$ & $S$ & $D$ & $W$ & $T$ & $H_{1}$ & $H_{2}$ \\
\hline \hline Value $(\mathrm{mm})$ & 20 & 0.5 & 0.25 & 40 & 0.25 & 1 & 2 \\
\hline
\end{tabular}




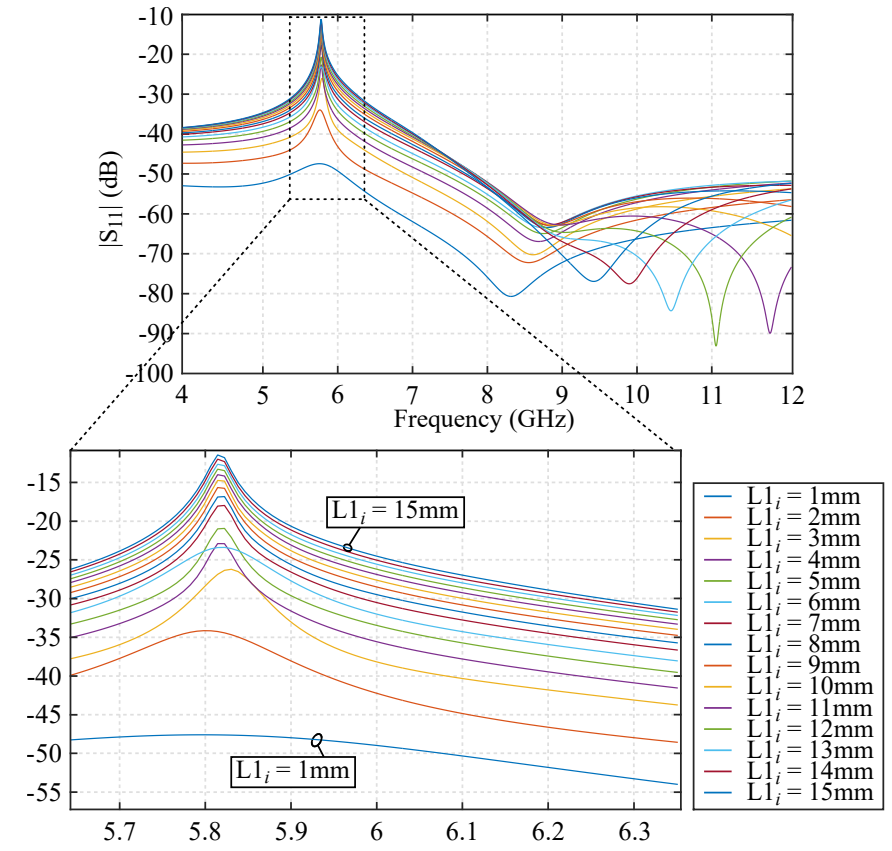

Fig. 7. Simulated $\left|S_{11}\right|$ parameter corresponding to the $H_{1 \_ \text {Block }}$ block, for different lengths, and detailed magnification.

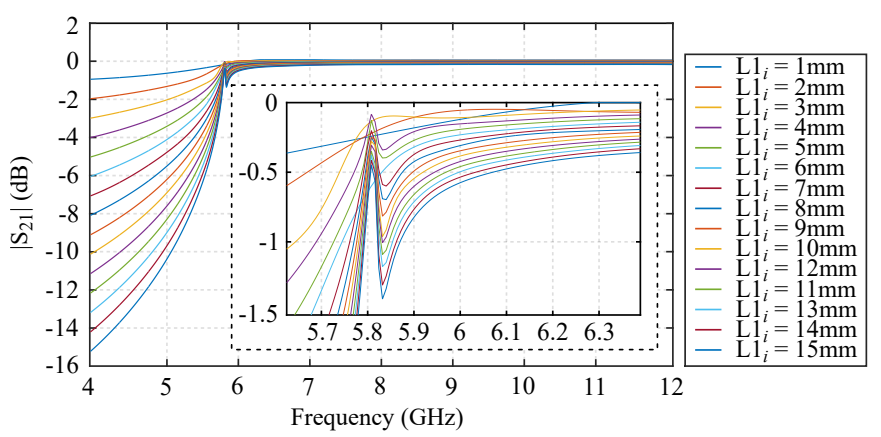

Fig. 8. Simulated $\left|S_{21}\right|$ parameter corresponding to the $H_{1 \_ \text {Block }}$ block, for different lengths, and detailed magnification.

model similar to the one previously presented in Figure 5a has been implemented as shown in Figure 12. The goal of the optimisation has been configured as follows: the frequency response is a passband in a frequency range between 8 and $9 \mathrm{GHz}$ and a $\left|\mathrm{S}_{21}\right|<-25 \mathrm{~dB}$ for the frequencies below and above the passband.

Once the optimisation has converged, the resulting variables v1 $\_i$ and $\mathrm{v} 2 \_i$ does not present integer values due to the interpolation between non previously calculated scattering parameters. Due to the fact that the variables $\mathrm{v} 1 \_i$ and $\mathrm{v} 2 \_i$ are related to the lengths of the segments of the SIW structure (each optimal value corresponds to half the length of the segment), integer values are preferred. For this purpose, the optimal values of the variables $\mathrm{v} 1 \_i$ and $\mathrm{v} 2 \_i$ have been truncated. A tuning process has not been required due to the results fulfilled the requirements after truncating the variables. Table II summarises the truncated optimal values for the variables $\mathrm{v} 1 \_i$ and $\mathrm{v} 2 \_$.

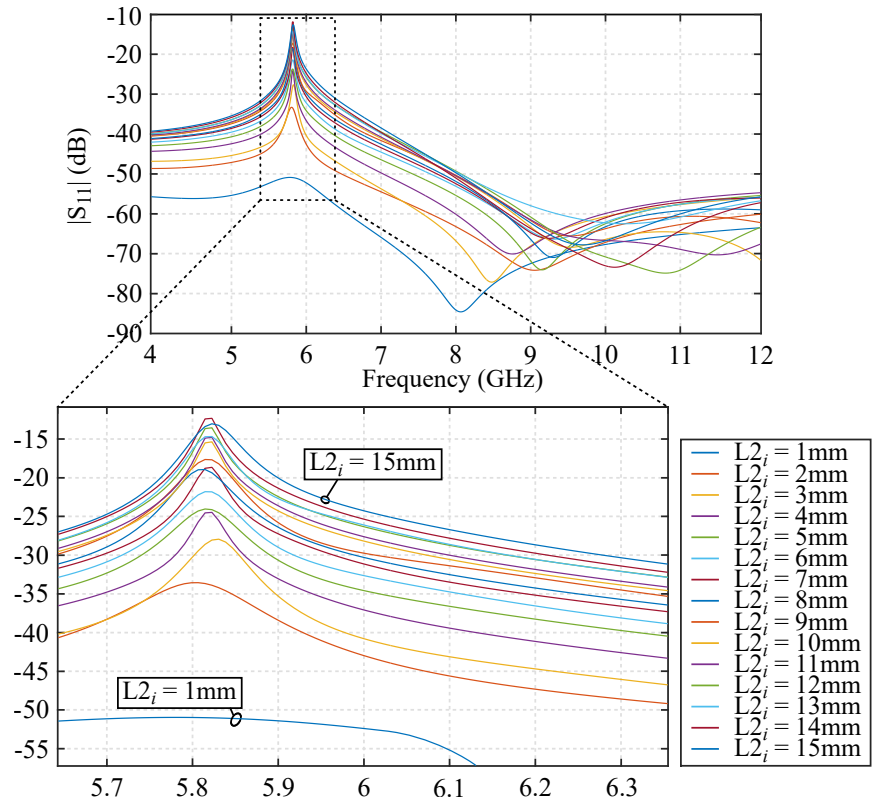

Fig. 9. Simulated $\left|S_{11}\right|$ parameter corresponding to the $H_{2}$ Block block, for different lengths, and detailed magnification.

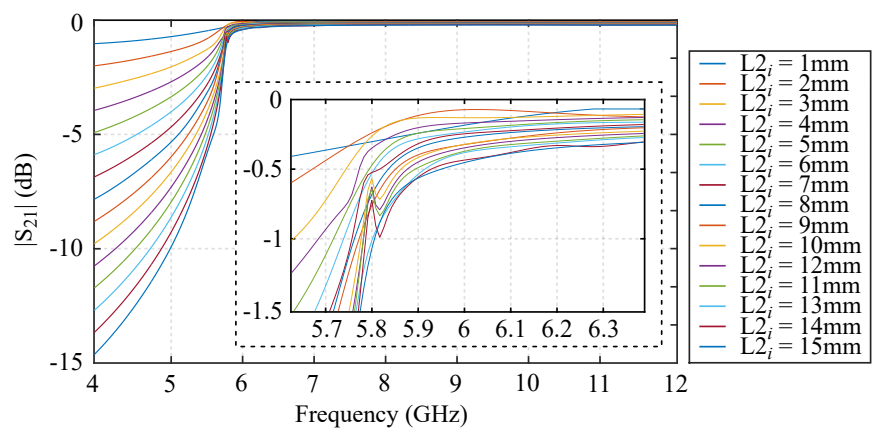

Fig. 10. Simulated $\left|S_{21}\right|$ parameter corresponding to the $H_{2}$ Block block, for different lengths, and detailed magnification.

As previously mentioned, in order to translate the values summarised in Table II into the lengths of the different blocks which conform the filter, multiplying by two the optimal values is required. Once the physical lengths of the blocks are calculated, a simplified notation to define the filter can be employed, which corresponds to the one already used in Figure

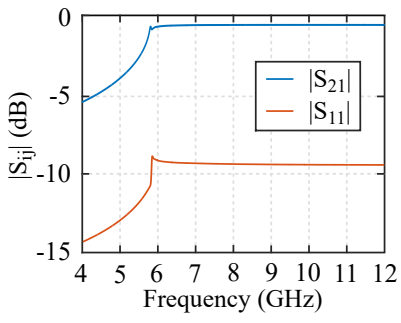

(a)

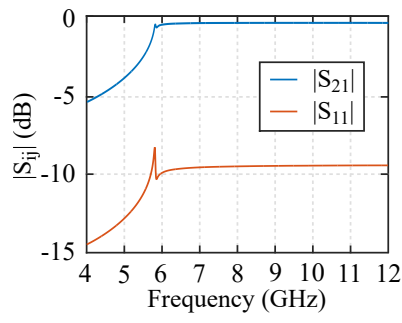

(b)
Fig. 11. Simulated $\left|S_{\mathrm{ij}}\right|$ parameters corresponding to the transition blocks. (a) $H_{1 \_2 \_B l o c k}$. (b) $H_{2 \_}{ }_{2}$ Block . 
TABLE II

TRUNCATED OPTIMAL VALUES FOR THE BPAHMSIW FILTER.

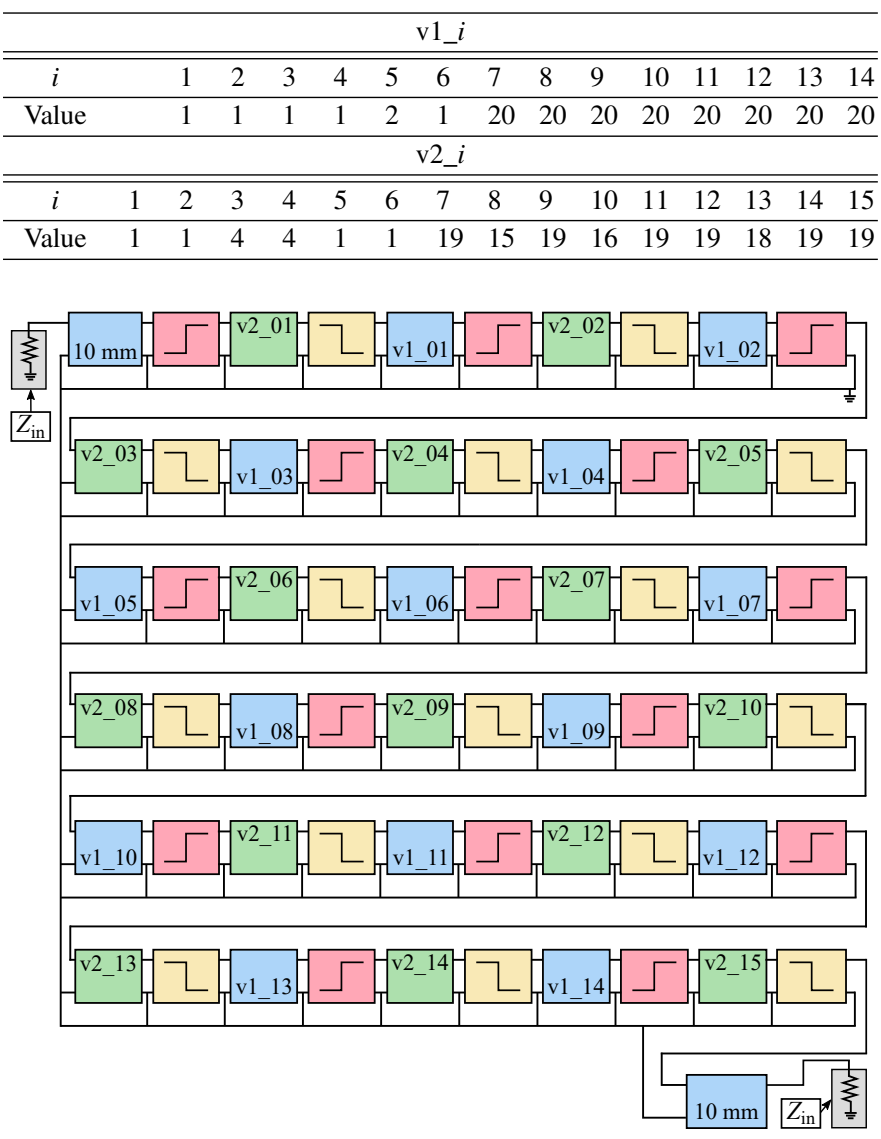

Fig. 12. Schematic model of the BPAHMSIW filter optimisation using ADS.

TABLE III

LENGTHS OF THE DIFFERENT SEGMENTS OF THE BPAHMSIW FILTER.

\begin{tabular}{|c|c|c|c|c|c|c|c|c|c|c|c|}
\hline \multicolumn{12}{|c|}{ Length $(\mathrm{mm})$ of the $i$-th segment whose height is $H_{1}$} \\
\hline$i$ & & 1 & 2 & 3 & 4 & 5 & 6 & 7 & 8 & 9 & 10 \\
\hline Length & & 5.5 & 5.5 & 5.5 & 5.5 & 6 & 5.5 & 15 & 15 & 15 & 15 \\
\hline$i$ & 11 & 12 & 13 & 14 & & & & & & & \\
\hline Length & 15 & 15 & 15 & 15 & & & & & & & \\
\hline \multicolumn{12}{|c|}{ Length $(\mathrm{mm})$ of the $i$-th segment whose height is $\mathrm{H}_{2}$} \\
\hline$i$ & 1 & 2 & 3 & 4 & 5 & 6 & 7 & 8 & 9 & 10 & 11 \\
\hline Length & 5.5 & 5.5 & 7 & 7 & 5.5 & 5.5 & 14.5 & 12.5 & 14.5 & 13 & 14.5 \\
\hline $\bar{i}$ & 12 & 13 & 14 & 15 & & & & & & & \\
\hline Length & 14.5 & 14 & 14.5 & 14.5 & & & & & & & \\
\hline
\end{tabular}

1. In this figure, instead of using the lengths of the $H_{1 \_B l o c k}$ and $H_{2}$ Block blocks, the lengths of the segments corresponding to the different heights, $H_{1}$ and $H_{2}$, are employed. For this purpose, the parameter $L_{\mathrm{T}}$, previously defined, is required. As a result, the lengths of the segments whose heights are $H_{1}$ and $\mathrm{H}_{2}$, respectively, are summarised in Table III. Consequently, taking into account the first and last blocks in Figure 12, whose lengths have been fixed to $10 \mathrm{~mm}$, and the values shown in Table III, the BPAHMSIW is completely characterised. The resulting filter, whose total length is $L_{\text {total }}=341 \mathrm{~mm}$, presents

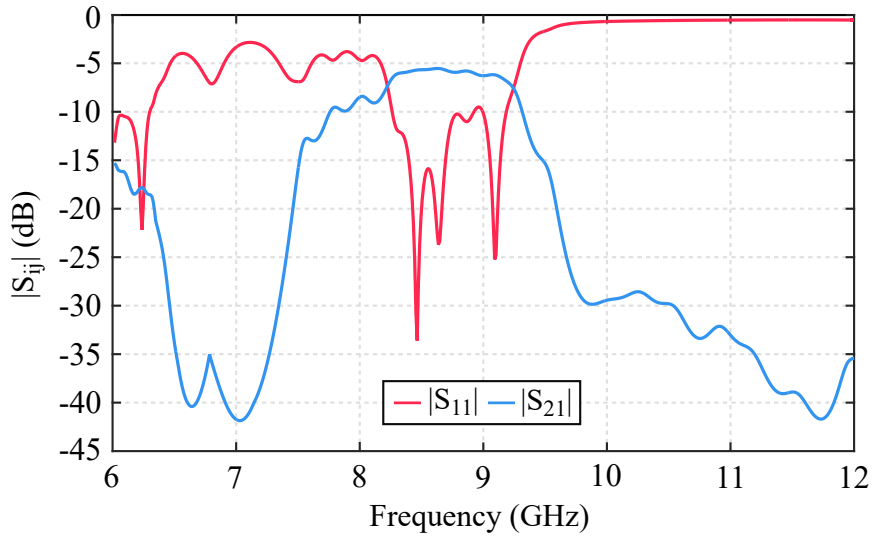

Fig. 13. Simulated $S$-parameters of the optimised BPAHMSIW filter.

TABLE IV

TRUNCATED OPTIMAL VALUES FOR THE BSAHMSIW FILTER.

\begin{tabular}{|c|c|c|c|c|c|c|c|c|c|}
\hline \multicolumn{10}{|c|}{$\mathrm{v} 1 \_i$} \\
\hline$i$ & & 1 & 2 & 3 & 4 & 5 & 6 & 7 & 8 \\
\hline Value & & 10 & 10 & 10 & 10 & 10 & 10 & 10 & 10 \\
\hline \multicolumn{10}{|c|}{$\mathrm{v} 2 \_i$} \\
\hline$i$ & 1 & 2 & 3 & 4 & 5 & 6 & 7 & 8 & 9 \\
\hline Value & 10 & 10 & 10 & 10 & 10 & 10 & 10 & 10 & 10 \\
\hline
\end{tabular}

the frequency response depicted in Figure 13. A $\left|S_{21}\right|=-6$ $\mathrm{dB}$ is achieved in the passband of the filter due to its length.

\section{B. Design of a BSAHMSIW filter}

A bandstop arbitrarily height-modulated textile integrated waveguide filter, denoted by BSAHMSIW filter, whose stopband belongs to the range of frequencies between 7.5 and $9 \mathrm{GHz}$, is proposed. The filter has been designed using the same methodology presented for the BPAHMSIW filter. For this reason, the MDIF files employed for the previous example have not been modified, and the new schematic model, composed of 37 blocks, following an structure analogous to the one previously presented in Figure 12.

In this example, the goal of the optimisation has been defined in order to obtain a frequency response with the following requirements: a stopband in a frequency range between 7.5 and $9 \mathrm{GHz}$ and $a\left|\mathrm{~S}_{21}\right|>-6 \mathrm{~dB}$ for the frequencies below and above the stopband. Once the optimisation has converged, the resulting variables $\mathrm{v} 1 \_i$ and $\mathrm{v} 2$ _ $i$ does not present the desired integer values. For this purpose, the optimal values of the variables $\mathrm{v} 1 \_i$ and $\mathrm{v} 2 \_i$ have been truncated and the tuning process has not been required due to the results fulfilled the requirements after truncating the variables. Table IV summarises the truncated optimal values for the variables $\mathrm{v} 1 \_i$ and $\mathrm{v} 2 \_i$.

As previously mentioned, in order to translate the values summarised in Table IV into the lengths of the different blocks which conform the filter, multiplying by two the optimal values is required. Once the physical lengths of the blocks are calculated and taking into account the previously defined 
TABLE V

LENGTHS OF THE DIFFERENT SEGMENTS OF THE BSAHMSIW FILTER.

\begin{tabular}{|c|c|c|c|c|c|c|c|c|c|c|}
\hline \multicolumn{11}{|c|}{ Length $(\mathrm{mm})$ of the $i$-th segment whose height is $H_{1}$} \\
\hline$i$ & & 1 & 2 & 3 & 4 & 5 & 6 & 7 & 8 & 9 \\
\hline Length & & 10 & 10 & 10 & 10 & 10 & 10 & 10 & 10 & 10 \\
\hline \multicolumn{11}{|c|}{ Length $(\mathrm{mm})$ of the $i$-th segment whose height is $\mathrm{H}_{2}$} \\
\hline$i$ & 1 & 2 & 3 & 4 & 5 & 6 & 7 & 8 & 9 & 10 \\
\hline Length & 10 & 10 & 10 & 10 & 10 & 10 & 10 & 10 & 10 & 10 \\
\hline
\end{tabular}

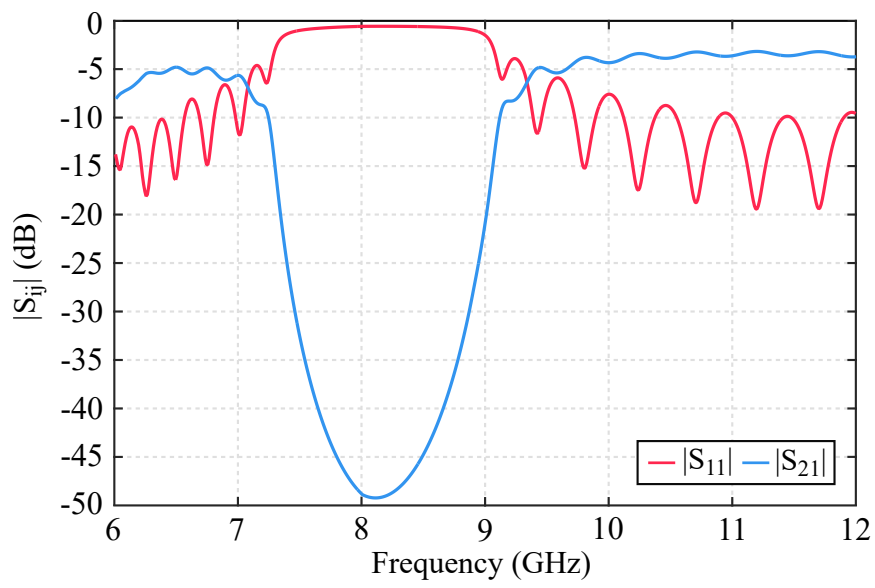

Fig. 14. Simulated scattering parameters of the optimised BSAHMSIW filter.

value of the $L_{\mathrm{T}}$ parameter, the lengths of the segments whose heights are $H_{1}=1 \mathrm{~mm}$ and $H_{2}=2 \mathrm{~mm}$, respectively, can be calculated as summarised in Table V.

Taking into account the first and last blocks of the filter, whose lengths have been fixed to $10 \mathrm{~mm}$, and the values shown in Table V, the BSAHMSIW is completely characterised. The resulting filter, whose total length is $L_{\text {total }}=204 \mathrm{~mm}$, presents the frequency response depicted in Figure 14.

\section{FABRICATION PROCEDURES}

The AHMSIW filters can be manufactured using 3Dprinting techniques. For this purpose, the structure of the filters must be divided in different parts. In the case of an air-filled filter, it is composed of two parts, as schematically represented in the top part of Figure 15. These parts are provided with the

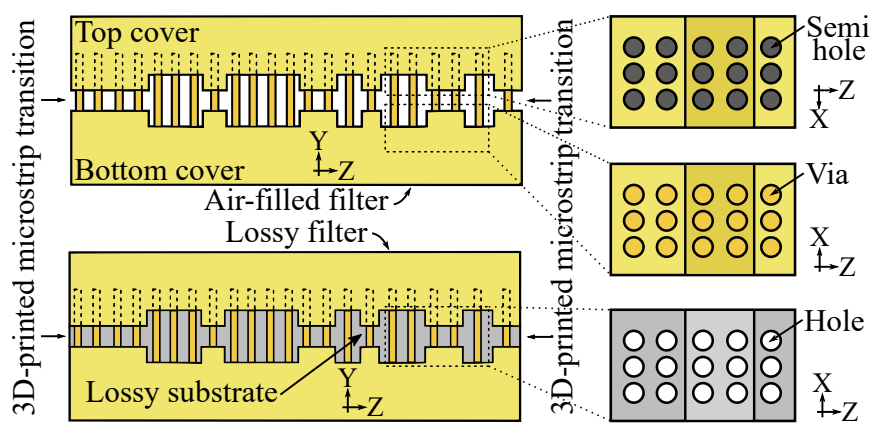

Fig. 15. Schematic overview of the different layers to 3D-print for the airfilled and lossy substrate-filled alternatives. vias or with the semi-holes, respectively. The inner parts of the filter and the vias will then be provided with the required conductivity through a thin gold layer thanks to a sputtering process.

Alternatively, the filter can be filled with a lossy substrate whose relative permittivity can be modified either by changing the printing material or by modifying the density of a previously selected material. This dielectric layer will be provided with holes in order to align and insert the conductive vias. Note that the substrate layer can be interchanged to modify the frequency of operation of the filter.

In both cases, the air-filled or the lossy substrate-filled filters, will be provided with a tapered 3D-printed microstrip to SIW transition, for their posterior experimental validation. These transitions can be manuactured separately from the filter and then connected to the filter structure using vias and holes. Additionally, this idea can be employed to 3D-print the filter structure in different pieces and achieve an adjustable structure.

\section{CONCLUSION}

A novel technique to design 3D-printable arbitrarily heihtmodulated SIW-based filters has been presented in this paper. This technique is based on simulating the scattering parameters of different SIW structures sweeping the corresponding lenghts, as well as obtaining the scattering parameters of two additional configurations of the SIW, based on an abrupt modification of the height. These scattering parameters are exported into the corresponding Touchtone files and then processed into three different MDIF files for their posterior use in ADS schematics for the optimisation of the filters.

Two different examples are additionally presented in this paper to demonstrate the validity of the technique. For this purpose, the development of both a bandpass and a bandstop filter are discussed. As a result, the simulated frequency responses of both filters fulfil the desired requirements. Additionally, the technique can be enhanced, although complicated, by using more than two different heights as presented in the examples.

The proposed filters can be manufactured using 3D-printing techniques and, therefore, this procedure has also been discussed in the paper. As a result, this technique allows the development of novel filters which can be provided with 3Dprinted SIW-to-microstrip transitions and integrated in planar structures.

\section{REFERENCES}

[1] S. Moscato et al., "Half-mode versus folded SIW filters: Modeling and design," presented in 2015 IEEE MTT-S Int. Conf. on Numerical Electromagnetic and Multiphysics Modeling and Optimization (NEMO), Ottawa, ON, 2015.

[2] H. Zhang et al., "Miniaturized Dual-Band SIW Filters Using E-Shaped Slotlines With Controllable Center Frequencies," in IEEE Microw. Wireless Components Lett., vol. 28, no. 4, pp. 311-313, Ap. 2018.

[3] K. Rabaani and N. Boulejfen, "Characteristic impedance and propagation constant assessment of Substrate Integrated Waveguide transmission line," presented in 11th Int. Design \& Test Symp. (IDT), Hammamet, Tunisia, Dec. 18-20, 2016. 